\title{
Dehydroepiandrosterone intake protects against 7,12-dimethylbenz(a)anthracene-induced mammary tumor development in the obese Zucker rat model
}

\author{
REZA HAKKAK $^{1,2,3}$, SAIED SHAAF ${ }^{1}$, CHAN-HEE JO $^{2}$, STEWART MacLEOD ${ }^{2}$ and SOHEILA KOROURIAN ${ }^{4}$ \\ Departments of ${ }^{1}$ Dietetics and Nutrition, and ${ }^{2}$ Pediatrics, University of Arkansas for Medical Sciences, 4301 W. Markham St.; \\ ${ }^{3}$ Arkansas Children's Hospital Research Institute, 13 Children's Way; ${ }^{4}$ Department of Pathology, University of \\ Arkansas for Medical Sciences, W. Markham St., Little Rock, AR 72205, USA
}

Received April 9, 2010; Accepted May 18, 2010

DOI: 10.3892/or_00000867

\begin{abstract}
Obesity has been epidemic in the US for over two decades; almost $65 \%$ of adults in the US are overweight. Obesity has been linked with the risk of development of various cancers, including breast cancer. Dehydroepiandrosterone (DHEA) is an over-the-counter dietary supplement used as an immunomodulating, anti-depressant, anti-aging, anti-cardiovascular disease, and anti-cancer agent and antiobesity supplement. The objectives of this study were to investigate the long-term effects of obesity and DHEA treatment on body weight gain and on 7,12-dimethylbenz(a)anthracene (DMBA)-induced mammary tumor development. Forty-three six-week-old obese female Zucker rats were used. Rats were randomly assigned and had ad libitum access to water and a diet of either chow (2016) as a control diet or chow with the addition of DHEA at a concentration of $6 \mathrm{~g} / \mathrm{kg}$ of chow as a DHEA diet. All rats were orally gavaged at age 50 days with $65 \mathrm{mg} \mathrm{DMBA} / \mathrm{kg}$ body weight. Rats were weighed and palpated twice weekly for detection of mammary tumors and sacrificed 155 days post-DMBA treatment. Obese rats fed the DHEA diet gained significantly less weight than obese control diet rats $(\mathrm{P}<0.001)$. At the end of the experiment, $55 \%$ of the control diet group developed mammary tumors, while no tumors were detected in the DHEA diet group
\end{abstract}

Correspondence to: Dr Reza Hakkak, Department of Dietetics and Nutrition, 4301 W. Markham St., Mail Slot 627, Little Rock, AR 72205, USA

E-mail: hakkakreza@uams.edu

Abbreviations: BMI, body mass index; DCIS, ductal carcinoma in situ; DHEA, dehydroepiandrosterone; DHEA-S, dehydroepiandrosterone sulfate; DMBA, 7,12-dimethylbenz(a)anthracene; DSHEA, Dietary Supplement and Health Education Act; IDC, invasive ductal carcinoma; IDP, intraductal proliferation; NHANES, National Health and Nutrition Examination Survey

Key words: obesity, Zucker rats, dehydroepiandrosterone, DMBAinduced mammary tumors
$(\mathrm{P}<0.001)$. Our results suggest that DHEA treatment can reduce body weight gain and protects against DMBA-induced mammary tumor development in the obese Zucker rat model.

\section{Introduction}

Breast cancer is the most common malignant tumor among women, and of all cancers, it is the second leading cause of cancer-related mortality in women in the US. In 2009, an estimated 192,370 women will be diagnosed with invasive breast cancer, and 40,170 women will eventually die from this disease (1).

Obesity has been an epidemic in the US for more than two decades, and the proportion of overweight and obese adults in the population continues to grow. Results from the 2005-2006 National Health and Nutrition Examination Survey (NHANES), using measured heights and weights, indicate that an estimated $32.7 \%$ of US adults 20 years and older are overweight, $34.3 \%$ are obese, and $5.9 \%$ are extremely obese $\left(B M I \geq 40 \mathrm{~kg} / \mathrm{m}^{2}\right)$ (2). Most countries are experiencing similar dramatic increases in obesity (3). Worldwide, more than one billion adults are overweight and over 300 million are obese. This trend has alarming health implications, as obesity is associated with serious health conditions, including type 2 diabetes, cardiovascular disease, and certain type of cancers, such as breast cancer (4).

A recent study investigated the role of overweight and obesity in carcinogenesis (5). This study not only documented an association between the BMI and mortality from several types of cancer, but also provided a reliable estimate of the contribution of overweight and obesity to the total mortality from cancer. They reported that women with the highest BMI of $40 \mathrm{~kg} / \mathrm{m}^{2}$ had death rates from all cancers combined that were $62 \%$ higher for women (with relative risk of death of 1.62) than the rates in women of normal weight. They reported a significant trend of increasing risk with higher BMI for death in women from cancers of the breast, uterus, cervix, and ovary (5).

Since the Dietary Supplement and Health Education Act (DSHEA) was passed in 1994, sales of dietary supplements have increased nearly 2-fold, from $\$ 8.8$ billion in 1994 to $\$ 14.7$ billion in 1999 (6). From 2000 to 2006, over \$21 billion 
is spent annually on dietary supplements, with sales increasing consistently by approximately $4 \%$ each year (7). Dehydroepiandrosterone (DHEA), a naturally occurring substance that is changed in the body to the hormones estrogen and testosterone, is a widely available over-the-counter dietary supplement used for weight loss. DHEA is also reported to have immunomodulating, anti-depressant, anti-aging, anticardiovascular disease, and anti-cancer effects, but considerably more evidence is required to substantiate these claims (8).

Zucker rats (fa/fa) are the best known, most widely used rat model for early onset obesity. Obesity in the Zucker rat is inherited as an autosomal recessive trait caused by a mutation in the leptin receptor gene (fa) $(9,10)$. Animals homozygous for the fa allele become noticeably obese by 3 to 5 weeks of age, and by 14 weeks of age their body composition is more than $40 \%$ lipid (11). The most valuable contribution of the Zucker rat has been its utility as a model of human earlyonset, hyperplastic-hypertrophic obesity. Many investigators have used this model to study the development, etiology, associated pathologies, possible treatment, and putative mechanisms of severe obesity (12).

We have shown previously that obesity increases the susceptibility to DMBA-induced mammary tumors (13). The main objective of this experiment was to investigate the effects of DHEA supplementation on DMBA-induced mammary tumor development using the obese Zucker rat model.

\section{Materials and methods}

Experimental design. All animal protocols were approved by the Institutional Animal Care and Use Committee (IACUC) at the University of Arkansas for Medical Sciences. Female obese Zucker rats (fa/fa) were purchased at 6 weeks of age from Harlan Industries (Indianapolis, IN). Rats were housed two per cage in the animal facilities at the Arkansas Children's Hospital Research Institute. Rats were randomly assigned to a diet of either chow (2016, Harlan Teklad, Madison, WI) as a control diet or chow with the addition of DHEA (Sigma Chemical Co., St. Louis, MO) at a concentration of $6 \mathrm{~g} / \mathrm{kg}$ of chow ( $0.6 \%$ by weight) as a DHEA diet. The 2016 diet contains minimal isoflavones and natural ingredients (corn, corn gluten meal, wheat, wheat middlings, soybean oil). Rats had ad libitum access to water and assigned diet. All rats were weighed twice weekly. At age 50 days, all rats received, via gavage, $65 \mathrm{mg}$ DMBA/kg body weight (Sigma Chemical Co.) in sesame oil. Beginning 4 weeks after DMBA treatment, all rats were palpated twice weekly for mammary tumor detection. The detection date and location of each mammary tumor were recorded for each rat. Rats were sacrificed 155 days post-DMBA treatment. All mammary tumors were excised and counted. Rats with tumor masses exceeding $2.5 \mathrm{~cm}$ in diameter were euthanized according to an IACUC-approved animal protocol. Sections of all tumors were placed in $10 \%$ neutral buffered formalin for histopathologic analysis. Sections $(4 \mu)$ of the paraffin-embedded tumors were stained with hematoxylin and eosin for light microscopic evaluation.

Pathology. A board-certified anatomic pathologist (SK) evaluated tumors in a blinded protocol. Tumors were classified as benign (essentially unremarkable breast parenchyma); intraductal proliferation (IDP), characterized by benign

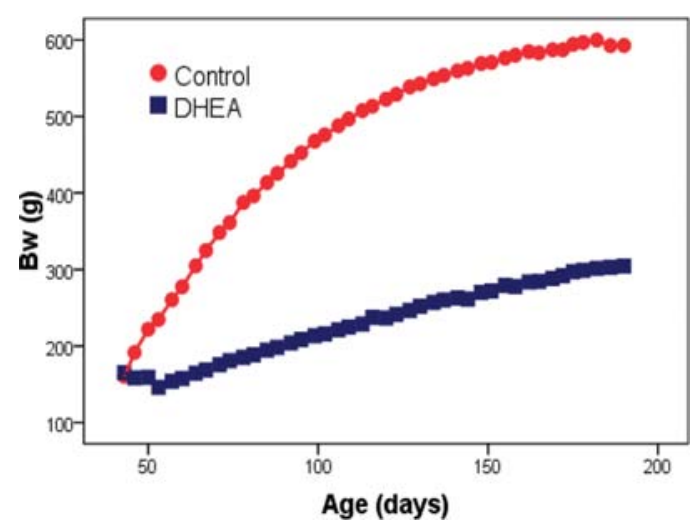

Figure 1. Body weights of control and DHEA-fed obese female Zucker rats.

Table I. Body weight (g) at different days of age during the experiment (mean $\pm \mathrm{SE})$.

\begin{tabular}{lcccc}
\hline Groups & Day 43 & Day 99 & Day 151 & Day 195 \\
\hline Control & $160 \pm 3$ & $468 \pm 9$ & $571 \pm 12$ & $593 \pm 12$ \\
DHEA & $165 \pm 3$ & $213 \pm 5^{\mathrm{a}}$ & $272 \pm 8^{\mathrm{a}}$ & $304 \pm 8^{\mathrm{a}}$
\end{tabular}

ap $<0.001$ (Mann-Whitney test).

epithelial proliferation or presence of ductal hyperplasia of the usual type, or multiple papillomas; ductal carcinoma in situ (DCIS); or invasive ductal carcinoma (IDC).

Statistical analysis. Mann-Whitney test was used to compare the body weight of the two groups. Kaplan-Meier analysis of tumor latency was performed. The log-rank test was used to compare median tumor-free times. Fisher's Exact test was used to compare percentage of rats with tumors and tumor histology in each group. Statistical significance was set at $\mathrm{P}<0.05$. For the few rats that were sacrificed early due to tumor burden, we assumed that the number of tumors remained constant until the end of the study. Data analyses were generated and plots were constructed using SPSS C version 17.0 for Windows (SPSS Inc., Chicago, IL) and SAS version 9.2 (SAS Inc., Cary, NC).

\section{Results}

Body weights. All rats gained weight during the course of the experiment (Fig. 1). The average body weights (mean \pm SE) at different ages during the experiment are shown in Table I. There were no differences in body weight of control and DHEAfed rats at the beginning of the experiment (age 43 days), but as the experiment continued the DHEA-fed rats gained less body weight than control rats $(\mathrm{P}<0.001)$ (Fig. 1 and Table I). The DHEA diet group showed a significant reduction in weight gain compared to the control diet rats $(\mathrm{P}<0.001)$.

Time course for tumor formation, latency, and multiplicity. The time course of palpable mammary tumor detection is shown in Fig. 2, and data are presented in Table II. The tumor 


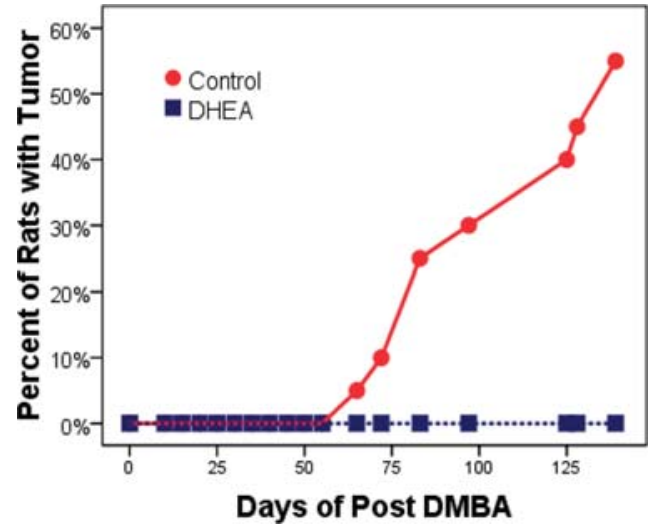

Figure 2. Mammary tumor incidence (percent of rats with tumors) of female rats (days post-DMBA treatment).

latency (the number of days post-DMBA treatment until the detection of the first mammary tumor) was shorter in control rats than the DHEA-fed group $(\mathrm{P}<0.001)$. The first mammary tumor detected in a control animal was 65 days post-DMBA treatment. In addition, $25 \%$ of the control rats had developed mammary tumors by 83 days post-DMBA treatment. By the end of the experiment, $55 \%$ of the control rats developed mammary tumors compared to no tumors detected in DHEAfed rats $(\mathrm{P}<0.001)$. The median number of mammary tumors per tumor-bearing rat (multiplicity) was compared. In regards to multiplicity, the obese control rats had a range of one to five tumors per rat compared to zero tumors in DHEA-fed rats (Table II).

Mammary tumor characteristics. Mammary tumor histology data are presented in Table II. A total of 18 mammary tumors were detected in the study. Table II shows the light microscopic classification of these mammary tumors. All of the 18 tumors were in the control diet group; no tumors were detected in the DHEA diet group. Pathologic analyses revealed tumors of the following classifications: 1 (6\%) benign, 2 (11\%) IDP, 11 (61\%) DCIS and 4 (22\%) IDC. Fig. 3 presents photomicrographs of tumors from each classification for this study.

\section{Discussion}

The major objectives of this study were i) to investigate the effects of DHEA and obesity on body weight gain and ii) to compare the effects of long-term DHEA intake and obesity on DMBA-induced mammary tumor development. In the present study, we found that i) obese DHEA-fed rats gained significantly less weight than obese control rats $(\mathrm{P}<0.001)$ and ii) $55 \%$ of DMBA-treated obese control rats developed mammary tumors compared to none of the DHEA-fed rats.

Obesity is associated with higher mortality and morbidity such as diabetes, cardiovascular disease, and several types of cancer including breast cancer. Several epidemiological and animal studies have shown the link between higher body weight and risk of breast cancer development (13-21). In fact, it has been shown that obese breast cancer patients, regardless of their menopausal status, have higher mortality and
A
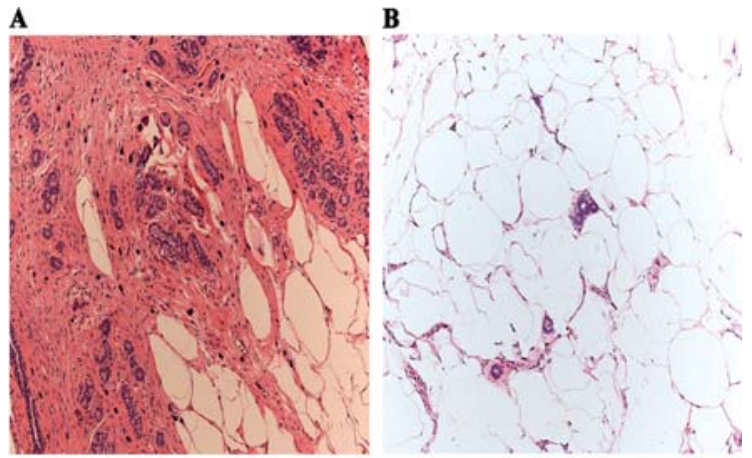

C



D

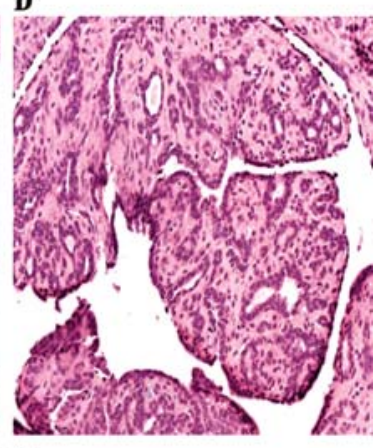

$\mathbf{E}$
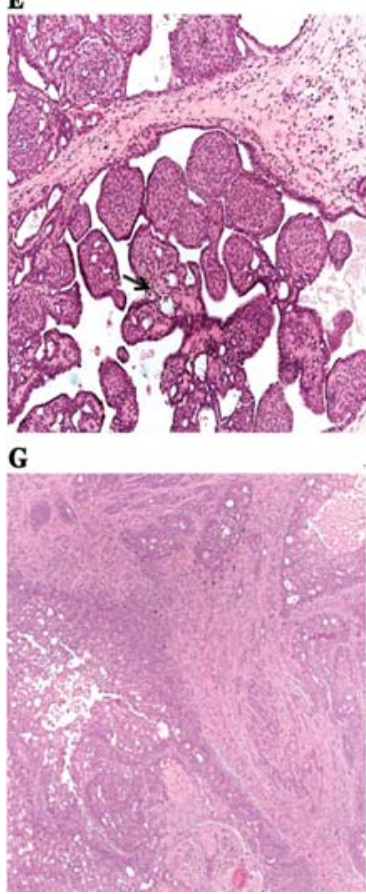

$\mathbf{F}$

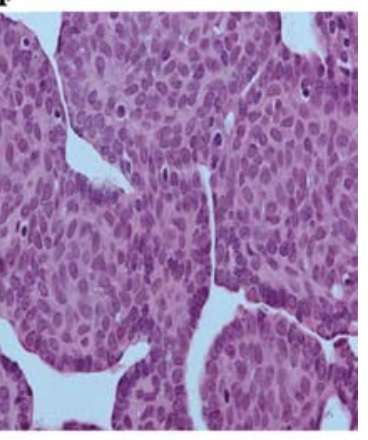

$\mathbf{H}$

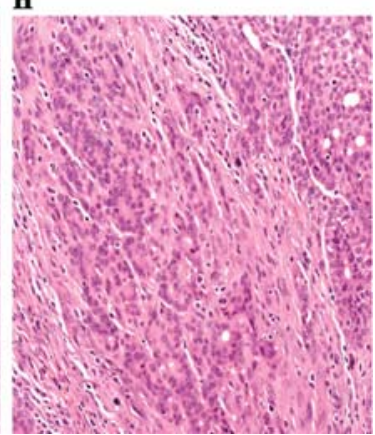

Figure 3. Mammary tumor histology. Normal breast tissue, original magnification, x10 (A and B): benign ductal and lobular structures were either surrounded by fibrous stroma or mature adipose tissue. No proliferation of epithelial cells noted in these images. Intraductal proliferation (IDP), papilloma, original magnification $\mathrm{x} 10(\mathrm{C})$; original magnification $\times 20$ (D). Ductal carcinoma in situ (DCIS), original magnification, x10 (E); original magnification, $x 40(F)$ : majority of ductal carcinomas showed micropapillary pattern. Arrow (E) shows an area within DCIS with numerous apoptotic bodies; note uniform proliferation of epithelial cells. Invasive ductal carcinoma (IDC), original magnification, $\mathrm{x} 4$ $(\mathrm{G})$; original magnification, $\mathrm{x} 40(\mathrm{H})$ : image shows infiltrating ductal carcinoma arising from a background of DCIS.

poorer outcome $(5,19,22-27)$. Also, obese breast cancer patients have higher tumor grade and tumor burden $(24,28)$. A recent study has evaluated the prognostic effects of obesity 
Table II. Characteristics of DMBA-induced mammary tumors in control and DHEA-fed obese female Zucker rats.

\begin{tabular}{|c|c|c|}
\hline & Control $(n=20)$ & DHEA $(n=23)$ \\
\hline \multicolumn{3}{|l|}{ Tumor onset } \\
\hline Day of first tumor ${ }^{\mathrm{a}}$ & 65 & No tumors present ${ }^{\mathrm{b}}$ \\
\hline Day at $25 \%$ tumors $^{\mathrm{c}}$ & 83 & No tumors present $t^{\mathrm{b}}$ \\
\hline \multicolumn{3}{|l|}{ Tumor incidence } \\
\hline$\%$ of rats with tumors ${ }^{\mathrm{c}}$ & 55 & $0^{\mathrm{b}}$ \\
\hline Multiplicity & $1(1-5)$ & 0 \\
\hline Total tumors $=18^{\mathrm{d}}$ & $18(100 \%)$ & $0^{\mathrm{b}}$ \\
\hline Benign & $1(6 \%)$ & 0 \\
\hline IDP & $2(11 \%)$ & 0 \\
\hline DCIS & $11(61 \%)$ & 0 \\
\hline IDC & $4(22 \%)$ & 0 \\
\hline
\end{tabular}

aPost-DMBA day at which the first mammary tumor was detectable by palpation. No tumors were detected in the DHEA-fed rats by the end


were detected in the DHEA-fed rats by the end of the experiment. ${ }^{\mathrm{d}}$ Number and percentage of total mammary tumors graded as benign, IDP, DCIS, or IDC as described in Materials and methods.

and weight change after breast cancer diagnosis and found obesity and weight change after diagnosis are inversely associated with breast cancer prognosis. The authors suggested that weight control is important among women with breast cancer (29).

Recently, our laboratory has shown that obesity is a major risk for breast cancer development using an obese Zucker rat DMBA-induced mammary tumor model. In these experiments, obese rats developed more mammary tumors at earlier time points compared to their lean littermates (13). In agreement with the present data, other studies using the obese Zucker rat model showed that treatment with DHEA resulted in reduced body weight gain (30-35). In laboratory studies, higher body weight has been associated with increases in both spontaneous and chemically induced mammary tumors in various strains of mice $(36,37)$.

The obesity pandemic is a very serious health problem, and obesity has been linked to breast cancer, especially in older women. Permanent weight reduction through diet and exercise is found to be difficult or impossible for most individuals (38). In regards to weight reduction and breast cancer development, a dietary supplement intervention that can help to reduce body weight and consequently reduce the risk of breast cancer development would be beneficial. Since DHEA is marketed as a supplement with purported health benefits, including weight loss, we used DHEA as a dietary supplement to investigate its effects on both body weight and its effects on breast cancer. The use of DHEA is controversial, with some reports indicating that DHEA may promote breast tumors in women $(39,40)$, while other reports suggest that DHEA will protect against breast cancer (41). One of the major goals of this investigation was to determine whether dietary supplementation with DHEA in obese Zucker rats would affect the development of DMBA-induced mammary tumors. To our knowledge, this report is the first to demonstrate that DHEA protects obese female Zucker rats against DMBA-induced mammary tumor development.

In the case of DHEA supplementation for anti-obesity, it is not known if DHEA decreases abdominal obesity in humans as has been shown in laboratory animal studies $(33,42-44)$. The results of epidemiologic studies of the relationship between DHEA and abdominal fat have been conflicting. In some studies, levels of serum DHEA or a DHEA metabolite, DHEA sulfate (DHEA-S), were significantly inversely related to abdominal obesity $(45,46)$, while in other studies in postmenopausal women, DHEA-S did not protect against obesity (47). In rats and mice, DHEA feeding reduces fat accumulation in both genetic- and diet-induced obesity models and has protective effects against insulin resistance induced by a high-fat diet $(33,42)$. Studies have reported an association between low levels of serum or urine DHEA or DHEA metabolites and increased breast cancer risk in premenopausal women (48-53). The relationship between endogenous androgens, such as DHEA, and breast cancer risk is less clear with both epidemiological and experimental data providing conflicting results. High serum DHEA-S has been linked to increased breast cancer risk in postmenopausal women (54). In postmenopausal women, obesity is predominantly abdominal and is reported to be positively associated with higher serum DHEA concentration $(45,46)$. In contrast, abdominal obesity has been associated with lower DHEA concentrations in premenopausal women (55). The reason for these differences is not clear, but several studies have shown abdominal obesity to be a marker for breast cancer risk in postmenopausal women (56).

Our present study has demonstrated that DHEA supplementation resulted in decreased weight gain in the obese rat model, as well as protection against DMBA-induced mammary tumors in DHEA-fed rats. A number of mechanisms may contribute to our observation that DHEA prevented mammary 
tumors in DMBA-treated obese Zucker rats. In the absence of estrogens, DHEA has been shown to have stimulatory effects on the growth of mammary tumors, due to the interaction of DHEA or its metabolites with the estrogen receptor. However, in the presence of estrogens, such as in intact Sprague-Dawley (non-obese) rats, DHEA has been reported to inhibit mammary tumor growth (57). DHEA also has chemopreventive activity against carcinogens such as DMBA, as well as UV-induced DNA damage. In a Sprague-Dawley model of DMBA-induced mammary tumors, DHEA treatment decreased the incidence of tumors, increased tumor latency, and significantly decreased tumor volume $(58,59)$.

DHEA has also been shown to inhibit the DMBAmediated induction of hepatic carcinogen activating enzymes, including cytochrome P450 1A1 (CYP1A1), CYP1A2, and CYP1B1 (60). These authors concluded that DHEA regulated the in vivo expression levels of these enzymes, decreased basal expression of CYP1A1 and CYP1A2, and reduced the induction of their expression by DMBA. This reduction in DMBA-activating activity may be a major factor in the chemopreventive activity exhibited by DHEA in our obese rat DMBA-induced mammary tumor model.

In conclusion, we have demonstrated that DHEA treatment can reduce body weight gain and protects against DMBAinduced mammary tumor development in the obese Zucker rat model. The mechanism for this protection remains to be defined and will be the subject of future investigation.

\section{Acknowledgements}

This study was supported by a grant from the Arkansas Biosciences Institute. We wish to thank Phaedra Yount and John Gregan for valuable assistance in preparation of this manuscript.

\section{References}

1. American Cancer Society. Cancer facts and figures. 2009.

2. National Health and Nutrition Examination Survey. 2009 National Center for Health Statistics. www.cdc.gov/NCCDPHP/ publications/AAG/pdf/obesity.pdf.

3. World Health Organization. WHO [www.who.int/nut/obs.htm].

4. Mokdad AH, Ford ES, Bowman BA, et al: Prevalence of obesity, diabetes, and obesity-related health risk factors, 2001. JAMA 289: 76-79, 2003

5.Calle EE, Rodriguez C, Walker-Thurmond $\mathrm{K}$ and Thun MJ: Overweight, obesity, and mortality from cancer in a prospectively studied cohort of U.S. adults. N Engl J Med 348: 1625-1638, 2003.

6. Wold RS, Lopez ST, Yau CL, et al: Increasing trends in elderly persons' use of nonvitamin, nonmineral dietary supplements and concurrent use of medications. J Am Diet Assoc 105: 54-63, 2005.

7. Nutrition Business Journal. Supplement business report 2006. San Diego, CA: New Hope Natural Media, Penton Media Inc, 2006.

8. Picciano MF and McGuire MK: Use of dietary supplements by pregnant and lactating women in North America. Am J Clin Nutr 89: S663-S667, 2009

9. Johnson MD, Bebb RA and Sirrs SM: Uses of DHEA in aging and other disease states. Ageing Res Rev 1: 29-41, 2002.

10. Zucker L and Zucker TF: Fatty, a new mutation in the rat. J Heredity 52: 275-278, 1961

11. Zucker TF and Zucker LM: Fat accretion and growth in the rat. J Nutr 80: 6-19, 1963.
12. Zucker LM: Fat mobilization in vitro and in vivo in the genetically obese zucker rat 'Fatty'. J Lipid Res 13: 234-243, 1972.

13. Hakkak R, Holley AW, MacLeod S, et al: Obesity promotes 7,12-dimethylbenz(a)anthracene-induced mammary tumor development in female zucker rats. Breast Cancer Res 7: R627-R633, 2005.

14. Cleary MP and Maihle NJ: The role of body mass index in the relative risk of developing premenopausal versus postmenopausal breast cancer. Proc Soc Exp Biol Med 216: 28-43, 1997.

15. Huang Z, Hankinson SE, Colditz GA, et al: Dual effects of weight and weight gain on breast cancer risk. JAMA 278: $1407-1411,1997$

16. Kwan ML, Kushi LH, Weltzien E, et al: Epidemiology of breast cancer subtypes in two prospective cohort studies of breast cancer survivors. Breast Cancer Res 11: R31, 2009.

17. McTiernan A: Associations between energy balance and body mass index and risk of breast carcinoma in women from diverse racial and ethnic backgrounds in the U.S. Cancer 88: 1248-1255, 2000.

18. Morimoto LM, White E, Chen Z, et al: Obesity, body size, and risk of postmenopausal breast cancer: The women's health initiative (United States). Cancer Causes Control 13: 741-751, 2002.

19. Reeves GK, Pirie K, Beral V, Green J, Spencer E and Bull D: Cancer incidence and mortality in relation to body mass index in the million women study: Cohort study. BMJ 335: 1134 2007.

20. Trentham-Dietz A, Newcomb PA, Storer BE, et al: Body size and risk of breast cancer. Am J Epidemiol 145: 1011-1019, 1997.

21. Wolk A, Gridley G, Svensson M, et al: A prospective study of obesity and cancer risk (Sweden). Cancer Causes Control 12: 13-21, 2001.

22. Barnett GC, Shah M, Redman K, Easton DF, Ponder BA and Pharoah PD: Risk factors for the incidence of breast cancer: Do they affect survival from the disease? J Clin Oncol 26: 3310-3316, 2008.

23. Barnett JB: The relationship between obesity and breast cancer risk and mortality. Nutr Rev 61: 73-76, 2003.

24. Berclaz G, Li S, Price KN, et al: Body mass index as a prognostic feature in operable breast cancer: The international breast cancer study group experience. Ann Oncol 15: 875-884, 2004.

25. Cleveland RJ, Eng SM, Abrahamson PE, et al: Weight gain prior to diagnosis and survival from breast cancer. Cancer Epidemiol Biomarkers Prev 16: 1803-1811, 2007.

26. Dal Maso L, Zucchetto A, Talamini R, et al: Effect of obesity and other lifestyle factors on mortality in women with breast cancer. Int J Cancer 123: 2188-2194, 2008.

27. Daling JR, Malone KE, Doody DR, Johnson LG, Gralow JR and Porter PL: Relation of body mass index to tumor markers and survival among young women with invasive ductal breast carcinoma. Cancer 92: 720-729, 2001.

28. Maehle BO, Tretli S, Skjaerven R and Thorsen T: Premorbid body weight and its relations to primary tumour diameter in breast cancer patients; its dependence on estrogen and progesterone receptor status. Breast Cancer Res Treat 68: 159169,2001

29. Chen X, Lu W, Zheng W, et al: Obesity and weight change in relation to breast cancer survival. Breast Cancer Res Treat (Internet). Jan 8, 2010.

30. Cleary MP: The antiobesity effect of dehydroepiandrosterone in rats. Proc Soc Exp Biol Med 196: 8-16, 1991.

31. Cleary MP, Fox N, Lazin B and Billheimer JT: A comparison of the effects of dehydroepiandrosterone treatment in zucker rats. Nutr Res 5: 1247-1257, 1985.

32. Cleary MP, Shepherd A and Jenks B: Effect of dehydroepiandrosterone on growth in lean and obese zucker rats. J Nutr 114: 1242-1251, 1984.

33. Cleary MP and Zisk JF: Anti-obesity effect of two different levels of dehydroepiandrosterone in lean and obese middle-aged female zucker rats. Int J Obes 10: 193-204, 1986.

34. Shepherd A and Cleary MP: Metabolic alterations after dehydroepiandrosterone treatment in zucker rats. Am J Physiol 246: E123-E128, 1984.

35. Svec F, Hilton CW, Wright B, Browne E and Porter JR: The effect of DHEA given chronically to zucker rats. Proc Soc Exp Biol Med 209: 92-97, 1995.

36. Waxler SH, Tabar P and Melcher LR: Obesity and the time of appearance of spontaneous mammary carcinoma in $\mathrm{C} 3 \mathrm{H}$ mice. Cancer Res 13: 276-278, 1953. 
37. Wolff GL, Kodell RL, Cameron AM and Medina D: Accelerated appearance of chemically induced mammary carcinomas in obese yellow (Avy/A) (BALB/c X VY) F1 hybrid mice. J Toxicol Environ Health 10: 131-142, 1982.

38. Brown KA and Simpson ER: Obesity and breast cancer: Progress to understanding the relationship. Cancer Res 70: 4-7, 2010.

39. Key T, Appleby P, Barnes I and Reeves G: Endogenous sex hormones and breast cancer in postmenopausal women: Reanalysis of nine prospective studies. J Natl Cancer Inst 94: 606-616, 2002.

40. Tworoger SS and Hankinson SE: Collection, processing, and storage of biological samples in epidemiologic studies: Sex hormones, carotenoids, inflammatory markers, and proteomics as examples. Cancer Epidemiol Biomarkers Prev 15: 1578-1581, 2006.

41. Labrie F: Future perspectives of selective estrogen receptor modulators used alone and in combination with DHEA. Endocr Relat Cancer 13: 335-355, 2006.

42. Hansen PA, Han DH, Nolte LA, Chen M and Holloszy JO: Dhea protects against visceral obesity and muscle insulin resistance in rats fed a high-fat diet. Am J Physiol 273: R1704-R1708, 1997.

43. Mohan PF, Ihnen JS, Levin BE and Cleary MP: Effects of dehydroepiandrosterone treatment in rats with diet-induced obesity. J Nutr 120: 1103-1114, 1990.

44. Yen TT, Allan JA, Pearson DV, Acton JM and Greenberg MM: Prevention of obesity in Avy/a mice by dehydroepiandrosterone. Lipids 12: 409-413, 1977.

45. Haffner SM, Valdez RA, Mykkanen L, Stern MP and Katz MS: Decreased testosterone and dehydroepiandrosterone sulfate concentrations are associated with increased insulin and glucose concentrations in nondiabetic men. Metabolism 43: 599-603, 1994.

46. Haffner SM, Valdez RA, Stern MP and Katz MS: Obesity, body fat distribution and sex hormones in men. Int J Obes Relat Metab Disord 17: 643-649, 1993.

47. Barrett-Connor E and Ferrara A: Dehydroepiandrosterone, dehydroepiandrosterone sulfate, obesity, waist-hip ratio, and noninsulin-dependent diabetes in postmenopausal women: The Rancho Bernardo Study. J Clin Endocrinol Metab 81: 59-64, 1996.

48. Helzlsouer KJ, Alberg AJ, Bush TL, Longcope C, Gordon GB and Comstock GW: A prospective study of endogenous hormones and breast cancer. Cancer Detect Prev 18: 79-85, 1994.
49. Kaaks R, Berrino F, Key T, et al: Serum sex steroids in premenopausal women and breast cancer risk within the European prospective investigation into cancer and nutrition (EPIC). J Natl Cancer Inst 97: 755-765, 2005.

50. Kabuto M, Akiba S, Stevens RG, Neriishi K and Land CE: A prospective study of estradiol and breast cancer in Japanese women. Cancer Epidemiol Biomarkers Prev 9: 575-579, 2000.

51. Rosenberg CR, Pasternack BS, Shore RE, Koenig KL and Toniolo PG: Premenopausal estradiol levels and the risk of breast cancer: A new method of controlling for day of the menstrual cycle. Am J Epidemiol 140: 518-525, 1994.

52. Thomas HV, Key TJ, Allen DS, et al: A prospective study of endogenous serum hormone concentrations and breast cancer risk in premenopausal women on the Island of Guernsey. Br J Cancer 75: 1075-1079, 1997.

53. Wysowski DK, Comstock GW, Helsing KJ and Lau HL: Sex hormone levels in serum in relation to the development of breast cancer. Am J Epidemiol 125: 791-799, 1987.

54. Dorgan JF, Stanczyk FZ, Longcope C, et al: Relationship of serum dehydroepiandrosterone (DHEA), DHEA sulfate, and 5androstene- 3 beta, 17 beta-diol to risk of breast cancer in postmenopausal women. Cancer Epidemiol Biomarkers Prev 6: 177-181, 1997.

55. Paolisso G, Ammendola S, Rotondi M, et al: Insulin resistance and advancing age: What role for dehydroepiandrosterone sulfate? Metabolism 46: 1281-1286, 1997.

56. Stoll BA: Obesity and breast cancer. Int J Obes Relat Metab Disord 20: 389-392, 1996.

57. Gatto V, Aragno M, Gallo M, et al: Dehydroepiandrosterone inhibits the growth of DMBA-induced rat mammary carcinoma via the androgen receptor. Oncol Rep 5: 241-243, 1998.

58. Li S, Yan X, Belanger A and Labrie F: Prevention by dehydroepiandrosterone of the development of mammary carcinoma induced by 7,12-dimethylbenz(a)anthracene (DMBA) in the rat. Breast Cancer Res Treat 29: 203-217, 1994.

59. Yang WS, Jeng CY, Wu TJ, et al: Synthetic peroxisome proliferator-activated receptor-gamma agonist, rosiglitazone, increases plasma levels of adiponectin in type 2 diabetic patients. Diabetes Care 25: 376-380, 2002.

60. Ciolino H, MacDonald C, Memon O, Dankwah M and Yeh GC: Dehydroepiandrosterone inhibits the expression of carcinogenactivating enzymes in vivo. Int J Cancer 105: 321-325, 2003. 